\title{
Lie Symmetries of Klein-Gordon and Schrödinger Equations
}

\author{
Muhammad Iqbal, Yufeng Zhang \\ College of Mathematics, China University of Mining and Technology, Xuzhou, China \\ Email: iqbalroghani85@yahoo.com,mathzhang@136.com
}

How to cite this paper: Iqbal, M. and Zhang, Y.F. (2018) Lie Symmetries of Klein-Gordon and Schrödinger Equations. Applied Mathematics, 9, 336-346. https://doi.org/10.4236/am.2018.93025

Received: February 29, 2018

Accepted: March 27, 2018

Published: March 30, 2018

Copyright () 2018 by authors and Scientific Research Publishing Inc. This work is licensed under the Creative Commons Attribution International License (CC BY 4.0).

http://creativecommons.org/licenses/by/4.0/

\begin{abstract}
In this paper, we investigate the Lie point symmetries of Klein-Gordon equation and Schrödinger equation by applying the geometric concept of Noether point symmetries for the below defined Lagrangian. Moreover, we organize a strong relationship among the Lie symmetries related to Klein-Gordon as well as Schrödinger equations. Finally, we utilize the consequences of Lie point symmetries of Poisson and heat equations within Riemannian space to conclude the Lie point symmetries of Klein-Gordon equation and Schrödinger equation within universal Riemannian space.
\end{abstract}

\section{Keywords}

Lie Symmetries of Klein-Gordon Equation, Lie Symmetries of Schrödinger Equation, Noether Point Symmetries of Conformal Lagrangian, $\operatorname{sl}(2, R)$ Algebra, Oscillator System

\section{Introduction}

According to Quantum Physics Klein-Gordon and Schrödinger equations are mainly two necessary equations, so it is compulsory that we can apply these equations and resolve their Lie point symmetries in the direction of discovering their invariant solutions by applying Lie point symmetries methods [1] [2] and [3]. As we know, Klein Gordon equation is an appropriate case of Poisson equation and Schrödinger equation is an important form of the heat equation. Since Lie point symmetries of Poisson and heat equations within Riemannian space have been studied in [4] [5], however in this paper we utilize these consequences to conclude the Lie point symmetries of Klein-Gordon equation and Schrödinger equation within universal Riemannian space.

One of the most important topics of the current study is the approach of conformally Lagrangians i.e. Lagrangians covered by the conformal transformation 
through potential as well as a metric guide in the equivalent equation; however, the dynamical variables are different. From every Lagrangian defining dynamical system, we determine a metric which is called kinematic metric defined by Lagrangian. Furthermore, Noether symmetries of the motion equations and conformal symmetries of that mentioned metric are a very strong connection. Moreover, the mentioned kinematic metric determined Laplace operator, and therefore the Lie point symmetries of Poisson equation have described the conditions related to conformal symmetries of kinematic metric. Since these conclusions are continued to Yamabe operator as well as investigate Lie point symmetries related to the conformal Klein-Gordon equation. We define the Lagrangian in the universal Riemannian space such as

$$
\mathcal{L}\left(x^{l}, x^{l}\right)=\frac{1}{2} f_{i j}\left(x^{l}\right) \dot{x}^{i} \dot{x}^{j}-W\left(x^{l}\right)
$$

And we can demonstrate with the intention of Noether symmetries of conformally Lagrangians be developed of metric $f_{i j}$ from the conformal algebra.

In this paper, we investigate the Lie symmetries of Klein-Gordon equation and Schrödinger equation by applying the geometric concept of Noether point symmetries for the above defined Lagrangian Equation (1); we organize a strong relationship among the Lie symmetries related to Klein-Gordon as well as Schrödinger equations. Finally, it will be demonstrated that if a Noether symmetry for the above Lagrangian Equation (1) is generated by the homothetic group element of kinematic metric then it further generates Lie symmetry for Klein Gordon equation and Schrödinger equation. We analyze that Noether point symmetries of Noether gauge are not a constant function.

We'll examine the case of the kinematic metric which introduces an Homothetic Vector (HV) or Killing Vector (KV) such that it produced Noether symmetries for classical Lagrangian Equation (1) and display that Lie point symmetry is a nonlocal symmetry in both cases of Klein Gordon equation. The information of Lie point symmetries of Klein Gordon equation and Schrödinger equation in a classical Riemannian space create achievable decisions of solutions of the above equations such that they are invariant by a mention Lie point symmetry. Furthermore, to study the Wheeler De Witt equation [6], they are used in Quantum Cosmology [7] [8] [9] [10] and [11] in a Riemannian space.

\section{Noether Point Symmetries Related to Conformal Lagrangian within Riemannian Paces}

Let us consider Lagrangian in a Riemannian space containing the metric $f_{i j}$ moving with the reaction of a potential $W\left(x^{l}\right)$, is given by

$$
\mathcal{L}=\frac{1}{2} f_{i j} \dot{x}^{i} \dot{x}^{j}-W\left(x^{l}\right) \text { where as } \dot{x}=\frac{\mathrm{d} x}{\mathrm{~d} t}
$$

According to the above reaction the equations of motion becomes

$$
R=\int \mathrm{d} t\left(\mathcal{L}\left(x^{l}, \dot{x}^{l}\right)\right)=\int \mathrm{d} t\left(\frac{1}{2} f_{i j} \dot{x}^{i} \dot{x}^{j}-W\left(x^{m}\right)\right)
$$


Now we shift the variable $t \rightarrow Z$ which is defined as

$$
\mathrm{d} z=M^{2}\left(x^{i}\right) \mathrm{d} t
$$

Now according to the new coordinates $\left(z, x^{l}\right)$ the equations of motion becomes

$$
R=\int \frac{\mathrm{d} z}{M^{2}\left(x^{l}\right)}\left(\frac{1}{2} f_{i j} M^{4}\left(x^{l}\right) x^{\prime i} x^{\prime j}-W\left(x^{l}\right)\right) \text { where as } x^{\prime i}=\frac{\mathrm{d} x}{\mathrm{~d} t}
$$

So the new Lagrangian becomes

$$
\mathcal{L}\left(x^{i}, x^{l}\right)=\frac{1}{2} M^{2}\left(x^{l}\right) f_{i j} x^{i} x^{\prime j}-\frac{W\left(x^{l}\right)}{M^{2}\left(x^{l}\right)}
$$

If we study the conformal transformation of a new potential function $\tilde{W}\left(x^{l}\right)=\frac{W\left(x^{l}\right)}{M^{2}\left(x^{l}\right)}$ and the new metric $\tilde{f}_{i j}=M^{2}\left(x^{l}\right) f_{i j}$, then according to the new coordinates $\left(z, x^{l}\right)$ the new Lagrangian becomes

$$
\tilde{\mathcal{L}}\left(x^{l}, x^{\prime l}\right)=\frac{1}{2} \tilde{f}_{i j} x^{\prime i} x^{\prime j}-\tilde{W}\left(x^{l}\right)
$$

Since the Lagrangian $\mathcal{L}$ in Equation (2) and the new Lagrangian $\mathcal{L}$ in Equation (7) of the same form therefore, the Lagrangian $\mathcal{L}\left(x^{l}, \dot{x}^{l}\right)$ ant the new Lagrangian $\mathcal{L}\left(x^{l}, x^{\prime}\right)$ is called conformal. Moreover, the reaction will be the same i.e. the equations of motion in the original coordinates $\left(t, x^{i}\right)$ as well as the latest coordinates $\left(z, x^{l}\right)$ under the Lagrangian $\mathcal{L}$ will be the same.

Corollary 2.1: In the conformably connected Lagrangians Equation (2) and Equation (7) the Noether point symmetries are included in the conformal algebra of $f_{i j}, \tilde{f}_{i j}$ metrics.

Lemma 2.2: For the two conformal Lagrangians transform the Euler Lagrange equations covariant the relating Lagrangians under the conformal transformation $\Leftrightarrow$ the Hamiltonian disappear.

Proof: Let us examine the Lagrangian $\mathcal{L}=\frac{1}{2} f_{i j} \dot{x}^{i} \dot{x}^{j}-W\left(x^{l}\right)$ containing the Euler Lagrange equations

$$
\ddot{x}^{i}+\Gamma_{j l}^{i} x^{i} \dot{x}^{j}+W^{, i}=0,
$$

whereas $\Gamma_{j l}^{i}$ called Christofell symbols for $i, j, l=1,2,3, \cdots, m$.

Now the equivalent Hamiltonian is of the form

$$
H=\frac{1}{2} f_{i j} \dot{x}^{i} \dot{x}^{j}+W\left(x^{l}\right)
$$

For the new Lagrangian $\tilde{\mathcal{L}}\left(x^{l}, x^{\prime l}\right)=\frac{1}{2} M^{2}\left(x^{l}\right) f_{i j} x^{\prime i} x^{\prime j}-\frac{W\left(x^{l}\right)}{M^{2}\left(x^{l}\right)}$ the produced Euler Lagrange equations as

$$
x^{\prime \prime i}+\hat{\Gamma}_{j l}^{i} x^{\prime i} x^{\prime j}+\frac{1}{M^{4}} W^{, i}-\frac{2 W}{M^{5}} W^{, i}=0
$$


whereas

$$
\hat{\Gamma}_{j l}^{i}=\Gamma_{j l}^{i}+(\ln M)_{, l} \pi_{j}^{i}+(\ln M)_{, l} \pi_{j}^{i}-(\ln M)^{, i} f_{i l}
$$

Now the equivalent Hamiltonian is of the form

$$
\tilde{H}=\mathrm{d} x^{i} \dot{x}^{i} \dot{x}^{j}+\frac{W\left(x^{l}\right)}{M^{2}\left(x^{l}\right)}
$$

Now to prove that these two equations are the same we using the conformal transformation

$$
x^{\prime i}=\frac{\mathrm{d} x^{i}}{\mathrm{~d} z}=\frac{\mathrm{d} x^{i}}{\mathrm{~d} t} \frac{\mathrm{d} t}{\mathrm{~d} z}=\dot{x}^{i} \frac{1}{M^{2}} \text { and } x^{\prime \prime i}=x^{. i} \frac{1}{M^{4}}-2 \dot{x}^{i} \dot{x}^{j}(\ln M)_{, j} \frac{1}{M^{4}}
$$

Now by substituting Equation (13) in Equation (10) we get

$$
\ddot{x}^{i} \frac{1}{M^{4}}-2 \dot{x}^{i} \dot{x}^{j}(\ln M)_{, j} \frac{1}{M^{4}}+\frac{1}{M^{4}} \Gamma_{j l}^{i} x^{\prime i} x^{\prime j}+\frac{1}{M^{4}} W^{, i}-\frac{2 W}{M^{5}} W^{, i}=0
$$

Furthermore, by substituting Equation (11) in Equation (14) we get

$$
\begin{aligned}
& \ddot{x}^{i}-2 \dot{x}^{i} \dot{x}^{j}(\ln M)_{, j}+\Gamma_{j l}^{i} x^{\prime i} x^{\prime l}+2(\ln M)_{, j} \dot{x}^{i} \dot{x}^{j} \\
& -(\ln M)^{, i} f_{i l} \dot{x}^{i} \dot{x}^{j}+W^{, i}-2 W(\ln M)^{, i}=0
\end{aligned}
$$

This follows that

$$
\ddot{X}^{i}+\Gamma_{j l}^{i} \dot{x}^{i} \dot{x}^{l}+W^{, i}-(\ln M)^{, i}\left(f_{i l} \dot{x}^{i} \dot{x}^{j}+2 W\right)=0
$$

Hence, the new Euler Lagrange Equation (15) correspond with the original Euler Lagrange Equation (8) $\Leftrightarrow f_{i l} \dot{x}^{i} \dot{x}^{j}+2 W=0$ i.e. the Hamiltonian vanishes. Obviously the converse is also true.

\section{Lie Symmetries Related to Klein-Gordon Equation}

According in the direction of Poisson equation $\Delta p-f\left(x^{i}, p\right)=0$ (where $\Delta=\frac{1}{\sqrt{|f|}} \frac{\partial}{\partial x^{i}}\left(\sqrt{|f|} f^{i j} \frac{\partial}{\partial x^{j}}\right)$ is called a Laplace operator) if we choose $f\left(x^{i}, p\right)=W\left(x^{i}\right) p$, then we get the Klein Gordon equation which is of the form

$$
\Delta p-W\left(x^{i}\right)=0
$$

Hence, by implementing the theorem which is stated as:

Theorem: Lie symmetries related to Poisson equation $\Delta p-f\left(x^{i}, p\right)=0$ of the $f_{i j}$ metric generated from the Conformal Killing Vectors (CKVs) describing the Laplace operator are the following

1) When $n>2$ then we get the vector as

$X=\phi^{i}\left(x^{l}\right) \partial_{i}+\left(\frac{2-n}{2} \xi\left(x^{l}\right) p+b_{0} p+c\left(x^{l}\right)\right) \partial_{p}$. Whereas $\phi^{i}\left(x^{l}\right)$ is called a $\mathrm{CKV}$ having the conformal factor $\xi\left(x^{l}\right)$ and $c\left(x^{l}\right)$ is a solution of $\Delta p-f\left(x^{i}, p\right)=0$ such as the following condition is satisfied

$$
\frac{2-n}{2} \Delta_{\xi p}+f^{i j} c_{, i j}-\phi^{l} f_{, l}-\frac{2-n}{2} \xi_{p} f_{, p}-\frac{2+n}{2} \xi g-c f_{, p}=0
$$


2) When $\ell=2$ then the Lie symmetry vector become $T=\phi^{i}\left(x^{l}\right) \partial_{i}+\left(b_{0} p+c\left(x^{l}\right)\right) \partial_{p}$. Whereas $\phi^{i}\left(x^{l}\right)$ is called a CKV having the conformal factor $\xi\left(x^{l}\right)$ and $c\left(x^{l}\right)$ is a solution of $\Delta p-f\left(x^{i}, p\right)=0$ such as the following condition is satisfied

$$
f^{i j} c_{, i j}-\phi^{l} f_{, l}-b_{0} p f_{, p}+\left(b_{0}-2 \xi\right) f-c f_{, p}=0
$$

Then we obtained a conclusion such as

Theorem 3.1: Lie symmetries related to Klein-Gordon equation Equation (16) of the $f_{i j}$ metric generated from the CKVs describing the Laplace operator are the following

1) When $n>2$ then we get vector as

$$
X=\phi^{i}\left(x^{l}\right) \partial_{i}+\left(\frac{2-n}{2} \xi\left(x^{l}\right) p+b_{0} p+c\left(x^{l}\right)\right) \partial_{p}
$$

whereas $\phi^{i}\left(x^{l}\right)$ is called a CKV having the conformal part $\xi\left(x^{l}\right)$ and $c\left(x^{l}\right)$ is a solution of Equation (16) such as the following condition is satisfied

$$
\phi^{l} W_{, l}+2 \xi W-\frac{2-n}{2} \Delta_{\xi}=0
$$

2) When $\ell=2$ then the Lie symmetry vector become

$$
X=\phi^{i}\left(x^{l}\right) \partial_{i}+\left(b_{0} p+c\left(x^{l}\right)\right) \partial_{p}
$$

whereas $\phi^{i}\left(x^{l}\right)$ is called a CKV having the conformal part $\xi\left(x^{l}\right)$ and $c\left(x^{l}\right)$ is a solution of Equation (16) such as the following condition is satisfied

$$
\phi^{l} f_{, l}+2 \xi W=0
$$

If we take the comparison between the Lagrangian Equation (2) and the Klein Gordon equation Equation (16) and the conformal factor holds the conditions $\xi_{; i j}=0$, for some important functions $\mathrm{CKV}$ or $\mathrm{KV}$ or $\mathrm{HV}$, then we analyze the following conclusion.

Proposition 3.2: When $n>2$, the Lie symmetries related to Klein-Gordon equation in favor of the $f_{i j}$ metric are connected to Noether symmetries of Lagrangian equation having the equivalent metric and potential which describe by the Laplace operator as follows

1) If a proper CKV or special CKV of the $f_{i j}$ metric, produce a Lie symmetries of Klein Gordon Equation (16) and also satisfy the condition $\Delta_{\xi}=0$, then it must generate a Noether symmetries of conformably Lagrangian Equation (2) so that the CKV convert into a HV or KV.

2) If an $\mathrm{HV}$ or $\mathrm{KV}$ of the $f_{i j}$ metric, produce Lie point symmetries related to Klein-Gordon Equation (16), subsequently the $f_{i j}$ metric, must generate Noether point symmetries related to Lagrangian Equation (2) with a constant gauge function.

\section{Lie Point Symmetries Related to Conformal Klein-Gordon Equation}

According to conformal Poisson equation $\tilde{L}_{f} p-\tilde{f}\left(x^{i}, p\right)=0$ (whereas $\tilde{L}_{f}$ is 
called the Yamabe operator) if we choose $\tilde{f}\left(x^{i}, p\right)=W\left(x^{l}\right) p$, then we get the conformal Klein Gordon equation (Yamabe KG) equation which is of the form

$$
\tilde{\mathcal{L}}_{f} p-W\left(x^{l}\right) p=0
$$

Hence, by implementing the theorem which is stated as

Theorem: Lie point symmetries related to conformal Poisson equation $\tilde{L}_{f} p-\tilde{f}\left(x^{i}, p\right)=0$ of the $f_{i j}$ metric generated from the CKVs describing the conformal Laplace operator as follows

$$
X=\phi^{i}\left(x^{l}\right) \partial_{i}+\left(\frac{2-n}{2} \xi\left(x^{l}\right) p+b_{0} p+c\left(x^{l}\right)\right) \partial_{p}
$$

whereas $\phi^{i}\left(x^{l}\right)$ is called a CKV having the conformal part $\xi\left(x^{l}\right)$ and $c\left(x^{l}\right)$ which is a solution of equation $\tilde{L}_{f} p-\tilde{f}\left(x^{i}, p\right)=0$ such as the following condition is satisfied

$$
f^{i j} c_{i ; j}-\phi^{l} g_{, l}-\frac{2-n}{2} \xi_{p} g_{p}-\frac{2+n}{2} \xi g-c\left(-N_{0} S+g_{p}\right)=0
$$

Then we obtained a conclusion such as

Theorem 4.1: Lie point symmetries related to conformal Klein-Gordon equation Equation (21) of the $f_{i j}$ metric generated from the CKVs describing Laplace operator are the following

1) When $n>2$ then we get vector as

$$
X=\phi^{i}\left(x^{l}\right) \partial_{i}+\left(\frac{2-\ell}{2} \xi\left(x^{l}\right) p+b_{0} p+c\left(x^{l}\right)\right) \partial_{p}
$$

whereas $\phi^{i}\left(x^{l}\right)$ is called a CKV having the conformal part $\xi\left(x^{l}\right)$ and $c\left(x^{l}\right)$ is a solution of Equation (21) such as the following condition is satisfied

$$
\phi^{l} W_{, l}+2 \xi W=0 \text { and } \tilde{\mathcal{L}}_{f} c-W d=0
$$

2) When $n=2$, then Equation (21) is called the Laplace Klein Gordon equation of Equation (16), and the Lie symmetry vector become

$$
X=\phi^{i}\left(x^{l}\right) \partial_{i}+\left(b_{0} p+c\left(x^{l}\right)\right) \partial_{p}
$$

whereas $\phi^{i}\left(x^{l}\right)$ is called a CKV having the conformal part $\xi\left(x^{l}\right)$ and $c\left(x^{l}\right)$ is a solution of Equation (16) such as the following condition is satisfied

$$
\phi^{l} g_{, l}+2 \xi W=0
$$

If we take the comparison between the Lagrangian Equation (2) and the conformal Klein Gordon equation Equation (21) and the conformal factor holds the conditions $\xi_{; i j}=0$, for some important functions $\mathrm{CKV}$ or $\mathrm{KV}$ or $\mathrm{HV}$, then we analyze the following conclusion.

\section{Proposition 4.2:}

1) If an $H V$ or $K V$ of the $f_{i j}$ metric, produce Lie point symmetries related to Klein-Gordon Equation (16), subsequently the $f_{i j}$ metric, must generate Noether point symmetries related to Lagrangian Equation (2) with a constant gauge function. 
2) If a proper CKV or special CKV of the $g_{i j}$ metric (dim $f_{i j} \geq 2$ ) which describe the conformal Laplace operator and produce Lie symmetries related to conformal Klein-Gordon Equation (21), subsequently it must generate a Noether symmetries of conformably Lagrangian Equation (2) so that the CKV convert into a $\mathrm{HV}$ or $\mathrm{KV}$.

\section{Lie Symmetries of Schrödinger Equation}

According to the heat equation $L(p)=v\left(t, x^{i}, p\right)$ if we choose $V(z, x, p)=W(x) p$, then we get Schrödinger equation which is of the form

$$
f^{i j} p_{i j}-\Omega^{i} p_{i}-p_{t}=W(x) p
$$

Theorem 5.1: Lie symmetries of Schrödinger equation Equation (24) by flux in $n$-dimensional Riemannian spaces be composed of homothetic algebra by $f_{i j}$ metric are the following

1) When $Z^{i}$ be non-gradient $\mathrm{KV}$ or an $\mathrm{HV}$

Then Lie symmetry vector become

$$
X=\left(2 b \xi t+b_{1}\right) \partial_{t}+b Z^{i} \partial_{i}+\left(b_{0} p+c(t, x)\right) \partial_{p}
$$

where $b_{1}, b$ are constant and $b_{0}, c\left(t, x^{l}\right), v\left(t, x^{l}, p\right)$ must satisfying the restriction equation

$$
L(c)-c W=0 \text { and } b \mathcal{L}_{z} W+2 \xi b W+b_{0}=0
$$

2) If $Z^{i}=L^{i}$ be the gradient $\mathrm{KV}$ or an $\mathrm{HV}$

Then the vector become

$$
X=\left(2 \xi \int T \mathrm{~d} t+b_{1}\right) \partial_{T}+T R^{, i} \partial_{i}+\left(\left(-\frac{1}{2} T_{, \mathrm{t}} R+G(t)\right) p+c(t, x)\right) \partial_{p}
$$

where $T(t), G(t), c\left(t, x^{l}\right), v\left(t, x^{l}, p\right)$ must satisfying the restriction equation

$$
L(c)-c W=0 \text { and } \mathcal{L}_{t} W+2 \xi W-\frac{1}{2} b^{2} L+e=0
$$

whereas the function $\mathrm{G}$ and $\mathrm{T}$ are calculated from the following results

$$
T_{, t t}=b^{2} T \text { and } \frac{1}{2} T_{, t} \xi+G_{, t}=d T
$$

If we take the comparison between the Lagrangian Equation (2) and the Schrödinger equation Equation (24), then we analyze the following conclusion.

Proposition 5.2: when an $H V I K V$ of the $f_{i j}$ metric, produces Lie symmetries related to Schrödinger equation Equation (24), then it must produce a Noether symmetries of Lagrangian Equation (2) with same metric $f_{i j}$ and same potential $W\left(x^{l}\right)$, obviously the converse also holds.

\section{Klein Gordon Equation and $s l(2, R)$ Algebra}

According to the preceding considerations for a constant gauge function, we proved that the Lie symmetries of Klein Gordon equation stimulate Noether 
symmetries used for the standard Lagrangian. Moreover, in this part, we study the case for a no constant gauge function while the stimulated Noether symmetries used for the standard Lagrangian, whereas, the stimulated Noether point symmetries to come from a comprehensive Lie point symmetries of Klein-Gordon equation. Obviously, if $\mathrm{HV}$ or KV produces Noether symmetries for the standard Lagrangian satisfy the condition i.e.

$$
W_{, l} Z^{l}+2 \xi_{t} W+b_{1}=0
$$

Then, it doesn't generate Lie symmetries for Klein Gordon equation. On the other hand, if a KV or HV generates Noether symmetries for the standard Lagrangian satisfy only the above condition go ahead to the two famous dynamical systems which are oscillator system and Kepler Ermakov system.

\section{Oscillator Systems}

We regard as the situation such that a metric acknowledge the gradient KV such that it produces Lie symmetries of the standard Lagrangian which can be written in the form as

$$
\mathrm{d} r^{2}=\mathrm{d} x^{2}+l_{B A} \mathrm{~d} y^{B} \mathrm{~d} y^{A}
$$

whereas, $R^{i}=\partial_{x}$ and $l_{B A}=l_{B A}\left(y^{b}\right)$ is called the tensor such that ordinary to the KV. According to the above coordinate, a Lagrangian can be written of the form

$$
\mathcal{L}=\frac{1}{2}\left(\dot{x}^{2}+l_{B A} \dot{y}^{B} \dot{y}^{A}\right)+W\left(x, y^{d}\right)
$$

Thus, for the gradient KV the Lie symmetry condition becomes

$$
W_{, x}+\lambda^{2} x=0
$$

The potential is follow as

$$
W\left(x, y^{d}\right)=-\frac{1}{2} \lambda^{2} x^{2}+G\left(y^{d}\right)
$$

The corresponding Noether symmetries in the form of vectors $\mathrm{e}^{ \pm \lambda z} \partial_{x}$, containing the measure function $g\left(z, x, y^{B}\right)=\lambda \mathrm{e}^{ \pm} x$, and the corresponding Noether integral is of the form

$$
I_{ \pm}=\mathrm{e}^{ \pm \lambda z} \dot{x} \mp \mathrm{e}^{ \pm \lambda z} X
$$

Now the Laplace Klein-Gordon equation described through the Equation (31) as well as Equation (33) is of the form

$$
p_{x x}+l^{B A} p_{B} p_{A}-\Pi^{B} p_{B}-\lambda^{2} x^{2} p-G\left(y^{d}\right) p=0
$$

Hence, the Equation (35) doesn't acknowledge a Lie symmetry for the common $l_{b A}, G\left(y^{b}\right)$, but, it is called independent through $x$ when a result be capable of in the type as $p\left(x, y^{B}\right)=v(x) R\left(y^{B}\right)$, means that the operator $\hat{I}=D_{x} D_{x}-\lambda^{2} x^{2}-I_{0}$ satisfies $\hat{I}_{p}=0$ i.e.

The Klein Gordon equation Equation (35) occupy Lie Bäcklund symmetry of [12] [13], containing the generator $T=\left(p_{x x}-\lambda^{2} x^{2}\right) \partial_{x}$. 
Regarding the conformal Klein Gordon equation such as

$$
p_{x x}+l^{B A} p_{B} p_{A}-\Pi^{B} p_{B}+\frac{(l-2)}{4(l-1)} S_{p}-\lambda^{2} x^{2} p-2 G\left(y^{d}\right) p=0
$$

Here for a KV $\phi^{b}$ we take $H_{\phi} S=0$, [14] therefore $S=S\left(y^{d}\right)$, so Equation (36) be able to write into shape of Laplace Klein-Gordon equation such that

$$
G\left(y^{d}\right)=2 G\left(y^{d}\right)-\frac{(l-2)}{4(l-1)} S\left(y^{d}\right) \text {. }
$$

\section{Kepler Ermakov Potential by Means of an Oscillator Term}

Suppose the gradient HV such that it generates Noether symmetry on behalf of a standard Lagrangian, then according to [15] [16] it is identified to acknowledge a gradient $\mathrm{HV}$, subsequently, we get the coordinates system such as

$$
\mathrm{d} r^{2}=\mathrm{d} s^{2}+s^{2} l_{B A} \mathrm{~d} y^{B} \mathrm{~d} y^{A}
$$

Here $\mathrm{HV}$ is $L^{i}=s \partial_{s},=1, H=\frac{1}{2} r^{2}$ and $l_{B A}=l_{B A}\left(y^{d}\right)$ is called tensor analytical normal.

With the help of above coordinates, required Lagrangian becomes

$$
\mathcal{L}=\left(\dot{r}^{2}+r^{2} l_{B A} y^{B} y^{A}\right) \frac{1}{2}-w\left(s, y^{d}\right)
$$

whereas gradient HV produces only Lie symmetries used for Ermakov potential comprehensive by the oscillator term, i.e.

$$
w\left(s, y^{d}\right)=-\frac{1}{2} \lambda^{2} s^{2}+\frac{G\left(y^{d}\right)}{r^{2}}
$$

The acknowledged Noether symmetries produce as of a gradient $\mathrm{HV}, L^{i}$ containing the vectors $X_{ \pm}=\frac{1}{\lambda} \mathrm{e}^{ \pm 2 \lambda t} \partial_{t} \pm \mathrm{e}^{ \pm 2 \lambda t} s \partial_{s}$, with equivalent gauge function $g\left(t, s, y^{B}\right)=\lambda \mathrm{e}^{ \pm 2 \lambda t} s^{2}$, related Noether Integral $\left(I_{+}=\frac{1}{\lambda} \mathrm{e}^{2 \lambda r} V-\mathrm{e}^{2 \lambda r} p p^{\prime}+\lambda \mathrm{e}^{2 \lambda r} p^{2}\right.$, $\left.I_{-}=\frac{1}{\lambda} \mathrm{e}^{-2 \lambda r} V-\mathrm{e}^{-2 \lambda r} p p^{\prime}+\lambda \mathrm{e}^{-2 \lambda r} p^{2}\right)$ and Hamiltonian 1 of Equation (38) it's easy to build the $1^{\text {st }}$ integral $\xi_{0}=l^{2}-I_{+} I_{-}$which depends on time which is of the form

$$
\xi_{0}=s^{4} l_{D A} \dot{y}^{B} \dot{y}^{A}+2 G\left(y^{d}\right) .
$$

Known as well-known Ermakov invariant [17] [18].

Laplace Klein-Gordon equation determine through Equation (39) as well as Equation (40) is given by

$$
p_{s s}+\frac{1}{s^{2}} l^{B A} p_{B A}+\frac{l-1}{s} p_{s}-\frac{1}{s^{2}} \Pi^{B} p_{B}+\lambda^{2} s^{2}+\frac{2}{s^{2}} G\left(y^{d}\right)=0
$$

Since the above equation doesn't introduce Lie symmetry, but it is separate in the logic such that $p\left(s, y^{d}\right)=v(s) R\left(y^{d}\right)$. So the operator becomes

$$
\xi=l^{B A} D_{B} D_{A}-\Pi^{B} D_{B}+2 G\left(y^{d}\right)-\xi_{0}
$$


This satisfies that $\xi_{p}=0$ i.e. introduce the Bäcklund symmetry containing the generator $X=\left(\xi_{p}\right) \partial_{p}$, [12] [13].

Regarding conformal Klein Gordon equation, the HV and Ricci scalar Equation (38) satisfy the situation $\mathcal{L}_{H} S+2 S=0$ [14], where $S=\frac{1}{s^{2}} \tilde{S}\left(y^{d}\right)$. After that, in the gradient $\mathrm{KV}$ we take up the expression $S\left(y^{b}\right)$ interested in a potential as well as obtained the similar solutions by means of Laplace Klein-Gordon equation. According to these results, it has been deduced with the purpose of generalized symmetries shift as of classical in the direction of quantum but Lie symmetries don't move.

\section{Conclusion}

In this paper, we apply the results obtained in the Lie point symmetries of Poisson and heat equations and study into universal Riemannian space the Lie point symmetries of Klein Gordon equation and Schrodinger equation. We acknowledge that how the Lie symmetries of Klein Gordon and Schrodinger equations are connected with Noether symmetries of the indicated Lagrangian and show that the kinematic metric is described by the standard Lagrangian. Finally, it will be demonstrated that if a Noether symmetry for the above Lagrangian equation (1) is generated by the homothetic group element of kinematic metric then it further generates Lie symmetry for Klein Gordon equation and Schrödinger equation. We analyze that Noether point symmetries of Noether gauge are not a constant function.

\section{Acknowledgements}

This work was supported by the National Natural Science Foundation of China (Grant No. 11371361), the Innovation Team of Jiangsu Province hosted by China University of Mining and Technology (2014), and the Key Discipline Construction by China University of Mining and Technology (Grant No. XZD 201602).

\section{References}

[1] Stephani, H. (1989) Differential Equations: Their Solutions using Symmetry. Cambridge University Press, New York.

[2] Ibragimov, N.H. (1985) Transformation Groups Applied to Mathematical Physics Translated from the Russian Mathematics and Its Applications (Soviet Series). D. Reidel Publishing Co., Dordrecht. https://doi.org/10.1007/978-94-009-5243-0

[3] Serge, L. (2010) Nonlinear Dynamics. Springer-Verlag, New York.

[4] Bozhkov, Y. and Freire, I.L. (2010) Special Conformal Groups of a Riemannian Manifold and Lie Point Symmetries of the Nonlinear Poisson Equation. Journal of Differential Equations, 249, 872-913. https://doi.org/10.1016/j.jde.2010.04.011

[5] Paliathanasis, A. and Tsamparlis, M. (2012) Lie Point Symmetries of a General Class of PDEs: The Heat Equation. Journal of Geometry and Physics, 62, 2443. https://doi.org/10.1016/j.geomphys.2012.09.004 
[6] De Witt, B.S. (1967) Quantum Theory of Gravity. I. The Canonical Theory. Physical Review, 160, 1113. https://doi.org/10.1103/PhysRev.160.1113

[7] Capozziello, S., De Laurentis, M. and Odintsov, S.D. (2012) Hamiltonian Dynamics and Noether Symmetries in Extended Gravity Cosmology. EPJC, 72, 2068.

https://doi.org/10.1140/epjc/s10052-012-2068-0

[8] Capozziello, S., De Ritis, R. and Scudellaro, P. (1994) Noether Symmetries in Quantum Cosmology. IJMPD, 3, 609. https://doi.org/10.1142/S0218271894000745

[9] Christodoulakis, T., Konas, G. and Papadopoulos, G.O. (2001) Conditional Symmetries and Phase Space Reduction towards gct Invariant Wave Functions, for the Class a Bianchi Type vi and vii Vacuum Cosmologies. Physics Letters B, 514, 149-154. https://doi.org/10.1016/S0370-2693(01)00793-6

[10] Vakili, B. (2008) Noether Symmetric f(r) Quantum Cosmology and Its Classical Correlations. Physics Letters B, 669, 209. https://doi.org/10.1016/j.physletb.2008.09.058

[11] Vakili, B. and Khazale, F. (2012) Noether Symmetric Classical and Quantum Scalar Field Cosmology. Classical and Quantum Gravity, 29, Article ID: 035015. https://doi.org/10.1088/0264-9381/29/3/035015

[12] Miller, J.R. (1983) The Technique of Variable Separation for Partial Differential Equations. Lectures Notes in Physics, 189, 184-208. https://doi.org/10.1007/3-540-12730-5_7

[13] Kamran, N. and McLenaghan, R.G. (1985) Separation of Variables and Symmetry Operators for the Conformally Invariant Klein-Gordon Equation on Curved Space-Time. Letters in Mathematical Physics, 9, 65. https://doi.org/10.1007/BF00398553

[14] Hall, G.S. and Steele, J.D. (1990) Conformal Vector Fields in General Relativity. Journal of Mathematical Physics, 32, 1847. https://doi.org/10.1063/1.529249

[15] Colley, A.A. and Tupper, B.O.J. (1989) Special Conformal Killing Vector Space-Times and Symmetry Inheritance. Journal of Mathematical Physics, 30, 2616. https://doi.org/10.1063/1.528492

[16] Daftardar, V. and Dadhich, N. (1994) Gradient Conformal Killing Vectors and Exact Solutions. General Relativity and Gravitation, 26, 859. https://doi.org/10.1007/BF02107144

[17] Lewis, H.R. (1967) Classical and Quantum Systems with Time-Dependent Harmonic-Oscillator-Type Hamiltonians. Physical Review Letters, 18, Article ID: 510512. https://doi.org/10.1103/PhysRevLett.18.510

[18] Moyo, S. and Leach, P.G.L. (2002) A Note on the Construction of the Ermakov Lewis Invariant. Journal of Physics A: Mathematical and General, 35, 5333.

https://doi.org/10.1088/0305-4470/35/25/312 